\title{
Interpretation of Sweep Frequency Response Analysis (SFRA) Traces for the Multiple Winding Faults which are Practically Simulated on the 10 KVA Power Transformer
}

\author{
Akshay A. Pandya ${ }^{1}$, Dr. B. R. Parekh ${ }^{2}$ \\ ${ }^{I}$ Research Scholar at SICART and Associate Professor, Department of Electrical Engineering, \\ Birla Vishvakarma Mahavidyalaya Engineering College, Vallabh Vidyanagar, Anand, INDIA \\ ${ }^{2}$ Prof. \& Head, Department of Electrical Engineering, Birla Vishvakarma Mahavidyalaya Engineering College, \\ Vallabh Vidyanagar, Anand, INDIA
}

\begin{abstract}
This paper presents how multiple winding faults can be detected by Sweep Frequency Response Analysis (SFRA). The test methods used by the authors for presenting the results are described. The Short Circuited Turns Fault on $H V$ " $V$ " phase winding and Earth Fault on $L V$ " $V$ " phase winding have been practically simulated simultaneously on the $10 \mathrm{KVA}, 11000 \mathrm{~V} / 440 \mathrm{~V}$, 3-phase, $50 \mathrm{~Hz}$, Dynll Power Transformer which is specially designed and developed in-house. The result of these simulated faults are presented and discussed. The SFRA data analysis is performed on three phase comparison basis and a conclusion has been drawn from it.
\end{abstract}

Keywords: Earth fault, Multiple winding fault, Power transformer, Short circuited turns fault, Sweep frequency response analysis.

\section{INTRODUCTION}

The SFRA is a powerful method for the detection and diagnosis of the defects in the active part of power transformers. It can deliver valuable information about the mechanical as well as electrical condition of core, windings, internal connections and contacts. No other single test method for the condition assessment of power transformers can deliver such a diversity of information. Therefore the SFRA is an increasingly popular test. The value of fingerprint data is more and more recognized by users all over the world. Comparing the time and frequency domain FRA test methods is seems to be obvious the SFRA, measuring directly in frequency domain, prevailed. Reproducibility is the key for a successful application of SFRA. [4]

Comparison with other diagnostic techniques show that the key advantages of FRA are its proven sensitivity to a variety of winding faults and a lesser dependency on previous reference measurements, but there is a need for an objective and systematic interpretation methodology.[1]

SFRA has been a key tool in the decision to scrap or reenergize a transformer. To get value from an SFRA test it is necessary to make sure that the measurements are credible, which requires good test technique and simple procedures; proper training is thus essential to control the measurement process. Subsequent interpretation of results is carried out with due respect to other tests and the context of the assessment - are we making SFRA measurements as part of a routine assessment, or as part of a post-fault investigation? Finally we should make conclusions which are supported by evidence and relate to individual components of the transformer phases, windings, tap changers etc. [5]

\section{DESCRIPTION OF THE TRANSFORMER}

The Power Transformer of 10 KVA which is specially designed and developed in-house as shown in Fig. 1 for studying and analyzing SFRA Traces by doing practical simulation of various transformer damages on it.

Some design details of the transformer may be found in Table 1

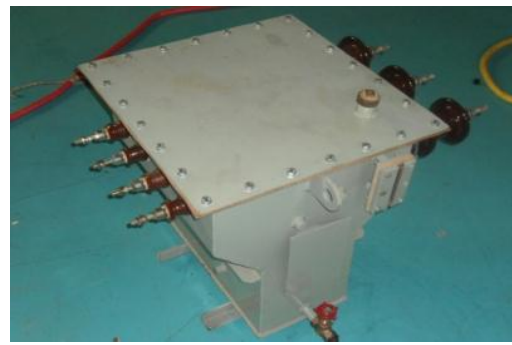

Fig. 110 KVA, 11000 V/440 V, 3-phase, 50 Hz, Dyn11 Power Transformer 
TABLE 1

BASIC DESIGN DETAIL FOR THE TEST TRANSFORMER

\begin{tabular}{|c|c|}
\hline Rated Power & $10 \mathrm{KVA}$ \\
\hline No. of Phases & 3 \\
\hline Rated Frequency & $50 \mathrm{~Hz}$ \\
\hline $\begin{array}{c}\text { Winding } \\
\text { Configuration }\end{array}$ & Dyn11 \\
\hline Voltage Rating & HV $-11000 \mathrm{~V}$ \\
LV $-440 \mathrm{~V}$
\end{tabular}

The HV delta connected winding has 10 nos. of uniform tapings where as LV star connected winding has no tapings.

Voltage \& No. of turns of HV Winding at each Tap is given in the Table 2.

TABLE 2

Voltage \& No. of turns of HV Winding at each Tap

\begin{tabular}{|c|c|c|c|}
\hline Tap No. & Volts & No. of Turns & Tapping Range \\
\hline 1 & 11688 & 8100 & $+6.25 \%$ \\
\hline 2 & 11550 & 8004 & $+5 \%$ \\
\hline 3 & 11413 & 7909 & $+3.75 \%$ \\
\hline 4 & 11276 & 7814 & $+2.5 \%$ \\
\hline 5 & 11139 & 7719 & $+1.25 \%$ \\
\hline 6 & 11000 & 7623 & $0 \%$ \\
\hline 7 & 10863 & 7528 & $-1.25 \%$ \\
\hline 8 & 10724 & 7432 & $-2.5 \%$ \\
\hline 9 & 10587 & 7337 & $-3.75 \%$ \\
\hline 10 & 10450 & 7242 & $-5 \%$ \\
\hline 11 & 10312 & 7146 & $-6.25 \%$ \\
\hline
\end{tabular}

Note : Tap 1 - Extreme Tap

Tap 6 - Normal Tap

Tap 11 - Lowest Tap

\section{TEST METHOD}

The main FRA test types are given below.

\subsection{End-to-end or End-to-end open}

In the "end-to-end open" test, the signal is applied to one end of each winding in turn, and the transmitted signal is measured at the other end. The magnetizing impedance of the transformer is the main parameter characterizing the low frequency response below first resonance using this configuration. This test is the more commonly used because of its simplicity and the possibility to examine each winding separately.

The "end-to-end open' tests can be made with the source applied on the phase terminal or on the neutral terminal. In principle, both should give similar results but the FRA user should specify the test set-up used and keep that information along with test data since it will influence the results.

\subsection{End-to-end short-circuit}

This test is similar to the end-to-end measurement above, but with a winding on the same being shortcircuited. Such measurements allow the influence of the core to be removed below about $10-20 \mathrm{KHz}$ because the low-frequency response is characterized by the leakage inductance instead of magnetizing inductance. The resonance at higher frequencies is similar to the one obtained using end-to-end measurement.

The short-circuited winding can be floating or grounded. For three-phase transformers, there are two levels of variations, either per-phase or three-phase short-circuit. Furthermore, the end-to-end short-circuit tests can be made with the source applied on the phase terminal or on the neutral terminal. This test can be made if there is an interest in obtaining information related to the leakage impedance at low frequency, or removing uncertainties related to analysis of the core influence when residual magnetism is present.

\subsection{Capacitive inter-winding}

The signal is applied to one end of a winding and the response is measured at one end of another winding on the same phase (not connected to the first one). By definition, this test is not possible between the series and common windings of auto transformers. The response using this configuration is dominated at low frequencies by the inter-winding capacitance. 


\subsection{Inductive inter-winding}

The signal is applied to a terminal on the HV side, and the response is measured on the corresponding terminal on the LV side, with the other end of both windings being grounded. The low frequency range of this test is determined by the winding turns ratio.

The choice of the list of the tests to perform should be adjusted depending on the purpose of the measurement.

To obtain benchmark test values for future comparison or for general condition assessment, the four main FRA test types presented above should be performed. If the test involves a winding with regulation (tap winding) the measurements should be performed at maximum tap. If there are limitations on the time available to perform all the tests, it is recommended to perform only the end-to-end tests on all windings. [1]

\section{Practical Simulation Of Multiple Winding Faults}

Authors have discussed here about the multiple winding faults, i.e. the short circuited turns fault on HV "V" phase winding and earth fault on LV "V" phase winding have been practically simulated simultaneously on the 10 KVA Power Transformer.

Before doing practical simulation of transformer damages, first SFRA Testing has been carried out on healthy condition of the transformer.

The SFRA Traces of this testing would be utilized as base line or previous data. The SFRA traces for the healthy condition of the transformer are shown in Fig. 2 to Fig. 4

Authors have performed total 9 test, End-to-end (6 tests) and End-to-end short-circuit (3 tests) on the power transformer at Tap 1 (Extreme Tap) for the healthy condition of the transformer.

Note : 1. For HV Delta connected winding, Red color indicates frequency response of U phase (1U 1V), Yellow color for $\mathrm{V}$ phase (1V $1 \mathrm{~W})$ and Blue color for $\mathrm{W}$ phase (1W 1U) in Figures 2, 3, 5, and 6.

2. For LV Star connected winding, Red color indicates frequency response of $U$ phase $(2 \mathrm{U} 2 \mathrm{~N})$, Yellow color for $\mathrm{V}$ phase $(2 \mathrm{~V} 2 \mathrm{~N})$ and Blue color for $\mathrm{W}$ phase $(2 \mathrm{~W} 2 \mathrm{~N})$ in Figures 4 and 7.

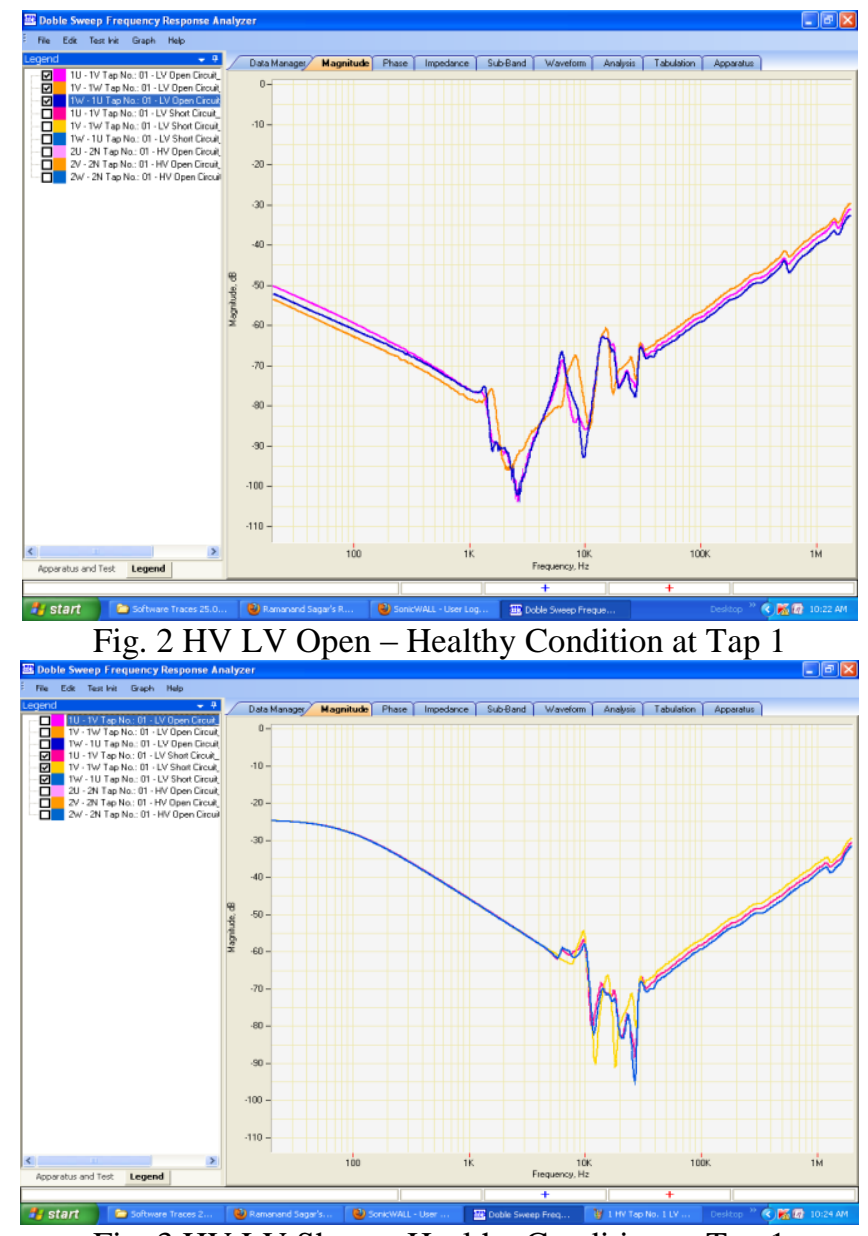

Fig. 3 HV LV Short - Healthy Condition at Tap 1 


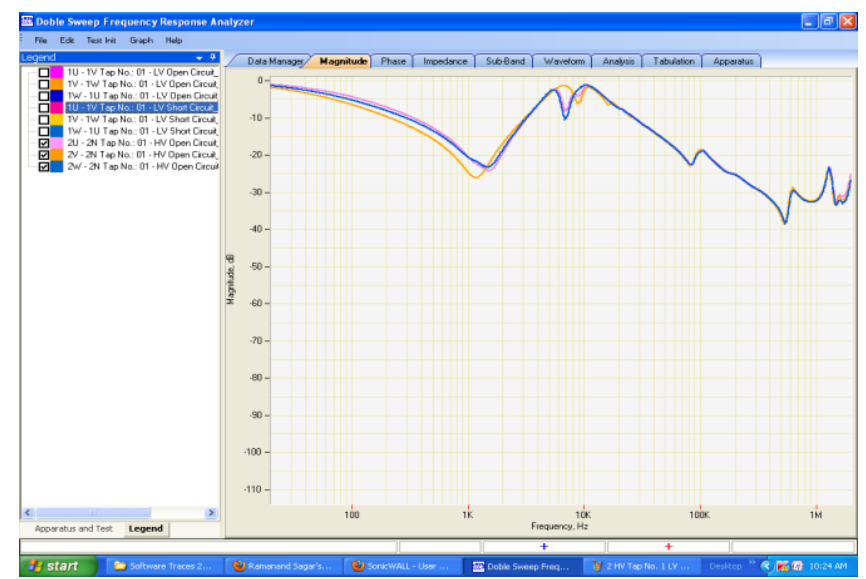

Fig. 4 LV HV Open - Healthy Condition at Tap 1

Authors have also performed total 9 test, End-to-end (6 tests) and End-to-end short-circuit ( 3 tests) on the power transformer at TAP 1 for the practically simulated multiple winding faults.

The short circuited turns fault on HV "V" phase winding (1V Ph Tap Short) and earth fault on LV "V" phase winding (2V EF) have been practically simulated simultaneously on the $10 \mathrm{KVA}$ Power Transformer.

The short circuited turns fault on HV "V" phase winding was applied practically as per the details given in Table 2, whereas the earth fault was applied practically at the terminal of LV "V" Phase winding of the power transformer.

TABLE 2

DETAILS OF PRACTICALLY SIMULATED SHORT CIRCUITED TURNS FAULT

\begin{tabular}{|c|c|}
\hline Total No. of Turns in HV Winding & 8100 \\
\hline $\begin{array}{c}\text { Due to HV Tapping Short } \\
\text { (Tap 4 \& 5 shorted) in "V" Phase }\end{array}$ & $\begin{array}{c}\text { 95 No. of Turns in HV "V" Phase } \\
\text { winding are getting shorted }\end{array}$ \\
\hline $\begin{array}{c}\text { \% of No. of Turns shorted in "V" } \\
\text { phase winding }\end{array}$ & $1.172 \%$ \\
\hline
\end{tabular}

The Combination of SFRA responses of all 9 tests are shown in Fig. 5 to 7.

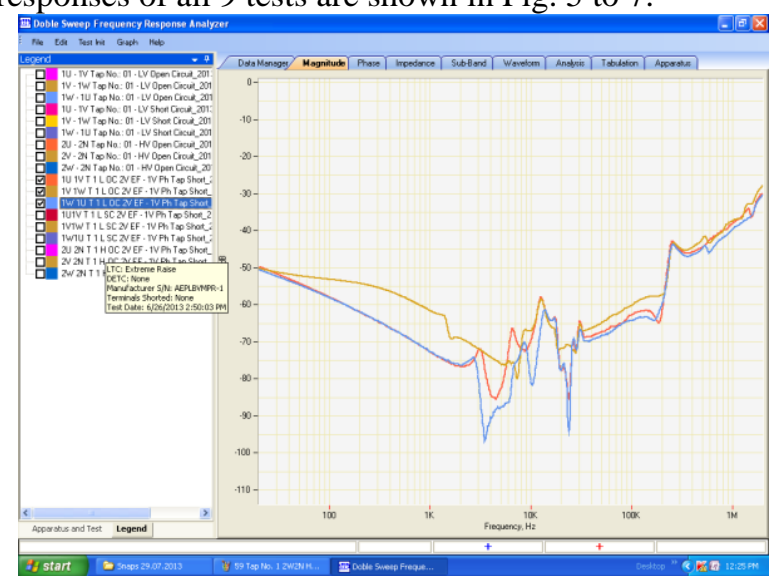

Fig. 5 Tap No. 1 HV LV Open 2V EF-1V Ph Tap Short

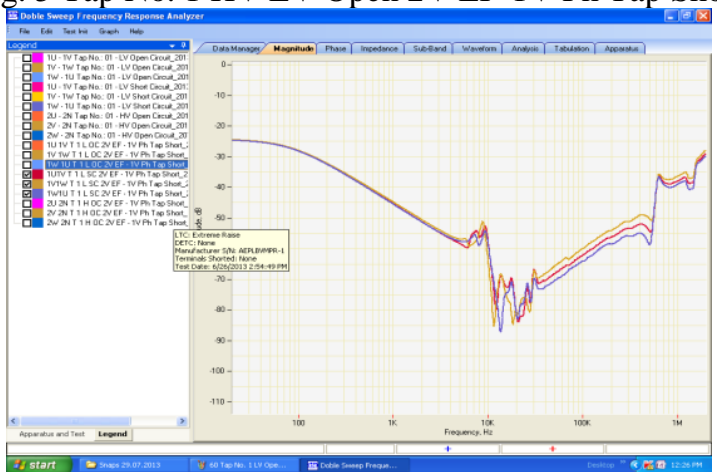

Fig. 6 Tap No. 1 HV LV Short 2V EF-1V Ph Tap Short 


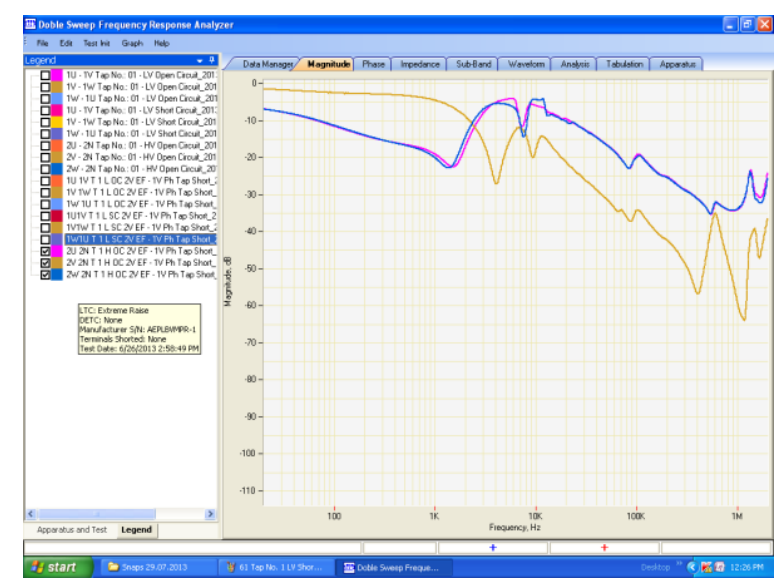

SFRA Data Analysis

Fig. 7 Tap No. 1 LV HV Open 2V EF-1V Ph Tap Short

Data analysis is performed on three phase comparison basis and following points are analyzed.

HV LV Open: Measured open circuit frequency responses of three phase of HV winding (11 KV, Delta) at Tap 1 are shown in Fig. 5

It is clear from the Fig. 5 that the response of $\mathrm{V}$ phase (1V 1W) (Middle Phase) is different than the outer phases and outer phases appear to be normal. In case of $\mathrm{V}$ phase winding the first resonance around $3.5 \mathrm{kHz}$ is absent. It can happen if the SFRA signal is getting internal short circuited path.

HV LV Short Circuit: Measured short circuit frequency responses of three phase of HV winding at Tap 1 are shown in Fig. 6

It is clear from the Fig. 6 that the short circuit responses of all the three phases of HV winding are similar and have general shape.

LV HV Open: Measured open circuit frequency responses of three phase of LV winding (440 V, Star) at Tap 1 are shown in Fig. 7

It is clear from the Fig. 7 that the response of $\mathrm{V}$ phase (2V 2N) (Middle Phase) is different than the outer phases and outer phases appear to be normal. The starting $\mathrm{dB}$ value of phase $\mathrm{V}$ is $-2 \mathrm{~dB}$ whereas starting $\mathrm{dB}$ value of other phases $(\mathrm{U} \& \mathrm{~W})$ is $-7 \mathrm{~dB}$ and the first resonance around $1.5 \mathrm{kHz}$ is absent in $\mathrm{V}$ phase response. The impedance value of phase $\mathrm{V}$ is lower in low frequency range (up to $1.5 \mathrm{kHz}$ ) and high in medium \& high frequency range as compared to the other phases $(\mathrm{U} \& \mathrm{~W})$. It can happen if the SFRA signal is getting earth fault path.

\section{Conclusion}

1. HV Winding: Based on the above SFRA data analysis it is concluded that the transformer has developed short circuited turns fault in $\mathrm{V}(1 \mathrm{~V} 1 \mathrm{~W})$ phase (i.e. V phase Tap short fault). It can be found from other conventional method like, No Load Excitation Current, Turns Ratio and Magnetic Balance test also.

2. When the first resonance in the low frequency range is absent in the open circuit frequency response of any phase of $\mathrm{HV}$ or LV winding and then it merges with the other open circuit frequency responses of two phases in the medium and high frequency range, than it indicates that the short circuited turns fault has been developed in that phase of that winding.

3. LV Winding: Based on the above SFRA data analysis it is concluded that the transformer has developed earth fault in $\mathrm{V}(2 \mathrm{~V} 2 \mathrm{~N})$ phase. It is possible to identify Earth Fault by other conventional method like, Tan Delta, Exciting Currents, Insulation Resistance, and TTR also.

4. When the open circuit frequency response of any phase of star connected winding shows that the impedance value of it is lower in low frequency range and high in medium \& high frequency range as compared to the other healthy phases than it indicates that the earth fault has been developed in that phase of star connected winding.

5. Based on the above SFRA data analysis it is concluded that the transformer has developed multiple winding fault, i.e. short circuited turns fault (i.e. Tap short fault) in $\mathrm{V}$ phase of $\mathrm{HV}$ winding and earth fault in $\mathrm{V}$ phase of LV winding. SFRA is able to identify both the faults very clearly which is not possible to achieve by only single test by any other method. It is the experience and interpretation of SFRA results plays a major role in identifying such multiple faults in transformer. 


\section{Acknowledgements}

The authors gratefully acknowledge the contributions of Mr. Tushar G. Patel and Mr. Snehal G. Mehta to the SMS Sabarmati, GETCO, Gujarat, INDIA

\section{REFERENCES}

[1] CIGRE Working Group A2.26, "Mechanical Condition Assessment of Transformer Windings Using Frequency Response Analysis (FRA)", Brochure 342, Paris, April 2008.

[2] Aradhana Ray, "Application of Signal Processing Techniques for Condition Monitoring of Electrical Equipment," Ph.D. thesis, Dept. of Electrical Engineering, Univ. M.S., College Faculty of Technology \& Engineering, September 2009.

[3] IEC 60076 - 18 Ed.1: Power Transformer Part - 18, "Measurement of Frequency Response", United Kingdom, July 2012.

[4] A. Kraetge, M. Kruger, J. L. Velasquez, H. Viljoen, A. Dierks, "Aspects of the Practical Application of Sweep Frequency Response Analysis (SFRA) on Power Transformer", $6^{\text {th }}$ Southern Africa Regional Conference CIGRE 2009.

[5] G. Matthew Kennedy, Charles Sweetser and Tony McGrail, "Field Experiences with Sweep Frequency Response Analysis", Doble Engineering Company $-73^{\text {rd }}$ Annual International Doble Conference, 2006

[6] IEEE PC 57.149 Guide for the Application and Interpretation of Frequency Response Analysis for Oil Immersed Transformer, March 2013 